\title{
Response to Letter to Editor "JAK2 V617F Mutation in Cervical Cancer Related to HPV \& STIs" by Stephen E. Langabeer
}

\author{
Amir Sohrabi \\ Department of Medical Epidemiology and Biostatistics, Karolinska Institutet, Stockholm, Sweden
}

\section{Dear Editor:}

We thank you for the opportunity to reply to the Langabeer's comments [1] on our previous manuscript [2]. The comments could be valuable for improving and clarifying some contents.

\section{Comments:}

Firstly, the JAK2 p.V617F (c.1849 G>T) is located in exon 12 (not exon 14) according to various literatures [3]. The acquired mutation can be presented in those patients with hematologic disorders. It appears that single nucleotide polymorphisms (SNPs) in immune mediators such as JAK2 could be associated with the risk of cervical cancer. However, these SNPs should be ascertained for justification in different communities and races with various genetic and epigenetic patterns [4].

The second argument raised by Langabeer [1] is that the RFLP method is an inefficient approach for identification of $G>T$ transversion. During the last decades, several PCR-based assays with different analytical sensitivity and specificity parameters have been developed for clinical and diagnostic applications. These include High Resolution Melting analysis, PCR-Amplification Refractory Mutation System, Reverse Transcription-PCR, direct DNA sequencing, Real Time PCR, PCR-RFLP, Allele Specific PCR, etc. However, they have their disadvantages besides advantages. Nowadays, next generation sequencing and other high throughput approaches with high accuracy and performance are applicable in SNPs studies, although they are limited by high cost and interpretations [5]. It seems that PCR-RFLP approach can be reliable in detecting SNPs as well as other methods in developing countries. The current study has been performed in Reference Health laboratory, MOHME, Tehran, Iran. The JAK2 mutation survey is a part of our current projects in SNPs analysis of cervical cancer subjects related to sexually transmitted infections. The PCR-RFLP has been validated and verified by use of approved positive controls. In addition, all of subjects' results have been confirmed by wild type, heterozygous and homozygous controls in each experiment.

We have been trying to decrease any false positive and negative results by utilizing high quality $C E$ and $I V D$ marked materials and equipment. Nonetheless, the statistical analysis on these subjects would be challenging due to some missing patients' data in that study. For improving and amending the analysis of SNPs survey, another manuscript is being prepared for better understanding of the JAK2 mutation associated with cervical malignancies as well as other potential polymorphisms in this issue. Obviously, more research is needed to achieve this endeavor for Cancer Care program.

\section{CONFLICTS OF INTEREST}

No potential conflicts of interest were disclosed. 


\section{REFERENCES}

1. Langabeer SE. "JAK2 V617F mutation in cervical cancer related to HPV \& STIs" - letter. J Cancer Prev 2019;24:59-60.

2. Abdolmaleki M, Sohrabi A. Characterization of JAK2 V617F (1849 $\mathrm{G}>\mathrm{T}$ ) mutation in cervical cancer related to human Papillomavirus and sexually transmitted infections. J Cancer Prev 2018;23:82-6.

3. Scott LM. The JAK2 exon 12 mutations: a comprehensive review.
Am J Hematol 2011;86:668-76.

4. Zhang Z, Fye S, Borecki IB, Rader JS. Polymorphisms in immune mediators associate with risk of cervical cancer. Gynecol Oncol 2014;135:69-73.

5. Didone A, Nardinelli L, Marchiani M, Ruiz ARL, de Lima Costa AL, Lima IS, et al. Comparative study of different methodologies to detect the JAK2 V617F mutation in chronic BCR-ABL1 negative myeloproliferative neoplasms. Pract Lab Med 2015;4:30-7. 